\author{
V. A. Lubarda · A. Marzani
}

\title{
Viscoelastic response of thin membranes with application to red blood cells: addendum
}

Received: 29 September 2009 / Published online: 25 November 2009

(C) The Author(s) 2009. This article is published with open access at Springerlink.com

In a recent paper [1] we presented a closed form solution to the problem of radial stretching of a viscoelastic hollow circular membrane under uniform tension applied over its internal boundary. The significance of this solution is that it can be used, in combination with the solution for a hollow membrane under uniform tension at both boundaries, to obtain the solution of a more general problem in which the membrane is loaded by different tensions at two boundaries. For example, in the analysis of the micropipette aspiration of the red blood cell, the tension on the internal boundary is induced by the micropipette suction pressure, while the tension on the external boundary is due to the internal pressure in the cell itself. Since this important generalization was not made in our original paper, we present it here, with a particular attention given to the loading combination in which the tension over the external boundary is adjusted, relative to the tension over the internal boundary, so that the entire membrane is under tensile state of stress, in both radial and circumferential directions, avoiding wrinkling due to the absence of its compressive strength.

If a hollow circular membrane, with the inner radius $R_{1}$ and the outer radius $R_{2}$, is under the tension $p_{1}=p+q$ over its internal boundary, and the tension $p_{2}=q$ over its external boundary, the radial and circumferential stresses in the membrane are $\sigma_{r}=\sigma+S$ and $\sigma_{\theta}=\sigma-S$, where

$$
\sigma=q-\frac{R_{1}^{2} p}{R_{2}^{2}-R_{1}^{2}}, \quad S=\frac{R_{1}^{2} R_{2}^{2}}{R_{2}^{2}-R_{1}^{2}} \frac{p}{r^{2}} .
$$

In the case of the viscoelastic constitutive model considered in [1], the isotropic and deviatoric strain components, $\epsilon=\left(\epsilon_{r}+\epsilon_{\theta}\right) / 2$ and $e=\left(\epsilon_{r}-\epsilon_{\theta}\right) / 2$, are governed by the differential equations

$$
\begin{aligned}
& t_{*} \dot{e}+e=\frac{R_{1}^{2}}{R_{2}^{2}-R_{1}^{2}} \frac{R_{2}^{2}}{r^{2}}\left(\frac{m}{2 \mu} p+\frac{t_{*}}{2 \mu_{0}} \dot{p}\right), \\
& \hat{t}_{*} \dot{\epsilon}+\epsilon=\frac{k}{2 K} \sigma+\frac{\hat{t}_{*}}{2 K_{0}} \dot{\sigma},
\end{aligned}
$$

where $\left(\mu, \mu_{0}\right)$ and $\left(K, K_{0}\right)$ are the shear and areal elastic moduli of the membrane, $m=1+\mu / \mu_{0}$, and $k=1+K / K_{0}$. The time-parameters are $t_{*}=\eta / \mu$ and $\hat{t}_{*}=\hat{\eta} / K$, where $\eta$ and $\hat{\eta}$ are the shear and areal coefficients of viscosity.

V. A. Lubarda $(\varangle)$

Department of Mechanical and Aerospace Engineering, University of California, San Diego,

La Jolla, CA 92093-0411, USA

E-mail: vlubarda@ucsd.edu

Tel.: 858-534-3169

Fax: 858-534-5698

A. Marzani

Department of Structural Engineering, University of Bologna, 40126 Bologna, Italy 
Suppose that $p=p(t)$ and $q=q(t)$ correspond to a suddenly applied tensions $p_{0}$ and $q_{0}$ at time $t=0$. The solutions of Eqs. (2) and (3) are then

$$
\begin{aligned}
& e(t)=\frac{p_{0}}{2 \mu} \frac{R_{1}^{2}}{R_{2}^{2}-R_{1}^{2}} \frac{R_{2}^{2}}{r^{2}}\left[m-\exp \left(-\frac{t}{t_{*}}\right)\right], \\
& \epsilon(t)=\frac{\sigma_{0}}{2 K}\left[k-\exp \left(-\frac{t}{\hat{t}_{*}}\right)\right],
\end{aligned}
$$

where $\sigma_{0}$ is defined by Eq. (1), with $(p, q)$ replaced by $\left(p_{0}, q_{0}\right)$. The corresponding radial displacement is $u=r(\epsilon-e)$, which gives

$$
u(r, t)=\frac{\sigma_{0} r}{2 K}\left[k-\exp \left(-\frac{t}{\hat{t}_{*}}\right)\right]-\frac{p_{0}}{2 \mu} \frac{R_{1}^{2}}{R_{2}^{2}-R_{1}^{2}} \frac{R_{2}^{2}}{r}\left[m-\exp \left(-\frac{t}{t_{*}}\right)\right] .
$$

In order that the membrane is under overall tensile state of stress, thus avoiding its wrinkling due to the absence of its compressive and buckling strengths, the circumferential stress has to be positive. Since $\sigma_{\theta}$ is a monotonically increasing function of $r$, a sufficient condition for this is that $\sigma_{\theta}\left(R_{1}, t\right) \geq 0$. In view of (1), this implies that

$$
q \geq \frac{R_{1}^{2}+R_{2}^{2}}{R_{2}^{2}-R_{1}^{2}} p, \quad \text { i.e., } \quad p_{2} \geq \frac{1}{2}\left(1+\frac{R_{1}^{2}}{R_{2}^{2}}\right) p_{1} .
$$

If the external tension $p_{2}$ is adjusted so that $\sigma_{\theta}\left(R_{1}, t\right)=0$, the equality sign holds in Eq. (7), and

$$
\sigma=\frac{R_{2}^{2} p}{R_{2}^{2}-R_{1}^{2}}
$$

In this case, the displacement expression (6) reduces to

$$
u(r, t)=\frac{p_{0}}{2 \mu} \frac{R_{2}^{2}}{R_{2}^{2}-R_{1}^{2}}\left\{\frac{\mu r}{K}\left[k-\exp \left(-\frac{t}{\hat{t}_{*}}\right)\right]-\frac{R_{1}^{2}}{r}\left[m-\exp \left(-\frac{t}{t_{*}}\right)\right]\right\} .
$$

The instantaneous and the long-term displacement responses are

$$
\begin{aligned}
& u\left(r, 0^{+}\right)=\frac{p_{0}}{2 \mu_{0}} \frac{R_{2}^{2}}{R_{2}^{2}-R_{1}^{2}}\left(\frac{\mu}{K_{0}} r-\frac{R_{1}^{2}}{r}\right), \\
& u(r, \infty)=\frac{p_{0}}{2 \bar{\mu}} \frac{R_{2}^{2}}{R_{2}^{2}-R_{1}^{2}}\left(\frac{\bar{\mu}}{\bar{K}} r-\frac{R_{1}^{2}}{r}\right),
\end{aligned}
$$

where

$$
\bar{\mu}=\frac{\mu \mu_{0}}{\mu+\mu_{0}}, \quad \bar{K}=\frac{K K_{0}}{K+K_{0}} .
$$

If the membrane is infinitely stiff to its areal change ( $\left.K, K_{0} \rightarrow \infty, \epsilon=0\right)$, Eqs. (9) and (10) reduce to

$$
\begin{aligned}
u(r, t) & =-\frac{p_{0}}{2 \mu} \frac{R_{1}^{2}}{R_{2}^{2}-R_{1}^{2}} \frac{R_{2}^{2}}{r}\left[m-\exp \left(-\frac{t}{t_{*}}\right)\right], \\
u\left(r, 0^{+}\right) & =-\frac{p_{0}}{2 \mu_{0}} \frac{R_{1}^{2}}{R_{2}^{2}-R_{1}^{2}} \frac{R_{2}^{2}}{r}, \quad u(r, \infty)=-\frac{p_{0}}{2 \bar{\mu}} \frac{R_{1}^{2}}{R_{2}^{2}-R_{1}^{2}} \frac{R_{2}^{2}}{r} .
\end{aligned}
$$

Finally, if $R_{2} \gg R_{1}$, (7) gives $q \geq p$ and $p_{2} \geq p_{1} / 2$, while (8) becomes $\sigma=p$. In this case, (9)-(13) simplify by replacing the ratio $R_{2}^{2} /\left(R_{2}^{2}-R_{1}^{2}\right)$ with 1 . 
Open Access This article is distributed under the terms of the Creative Commons Attribution Noncommercial License which permits any noncommercial use, distribution, and reproduction in any medium, provided the original author(s) and source are credited.

\section{Reference}

1. Lubarda, V.A., Marzani, A.: Viscoelastic response of thin membranes with application to red blood cells. Acta Mech. 202, $1-16(2009)$ 